\title{
Las enfermedades raras y su representación en la prensa española
}

\author{
María Teresa Santos Diez ${ }^{1}$ \\ Jesús Ángel Pérez Dasilva²
}

Recibido: 2018-04-17

Enviado a pares: 2018-04-18
Aprobado por pares: 2018-06-10

Aceptado: 2018-11-07

DOI: 10.5294/pacla.2019.22.1.10

\section{Para citar este artículo / to reference this article / para citar este artigo}

Santos, M. T. y Pérez, J. A. (2019). Las enfermedades raras y su representación en la prensa española. Palabra Clave 22(1), e22110. DOI: 10.5294/pacla.2019.22.1.10

\section{Resumen}

Este artículo es el resultado de una investigación centrada en la atención mediática que los diarios españoles El País, ABC, El Mundo y La Razón otorgan a las enfermedades raras (ER) desde el 1 de octubre de 2016 hasta el 30 de septiembre de 2017. Un tema de actualidad e interés social que contribuye a dar visibilidad a un grave problema de salud, que padecen menos de cinco personas por cada 10 000. Estas patologías se caracterizan por ser crónicas, potencialmente mortales y con un alto grado de discapacidad y dependencia. Utilizando una metodología de análisis de contenido se identifican las áreas temáticas y se cuantifica, analiza y compara el tratamiento realizado por los diarios mencionados. Los resultados muestran que es un tema emergente y de relevancia en la prensa nacional. Se trata de textos genéricos que se incluyen mayoritariamente en la sección de sociedad y que son elaborados con escasa profundización sin apenas explicaciones ni opiniones dado que solo utilizan una fuente. Las temáticas más recurrentes son medicina/enfermedades, investigación y tribunales. Por tanto, las ER están presentes en los diarios españoles y transmiten a la sociedad unan visión que se equipara al resto de enfermedades aportando un enfo-

1 orcid.org/0000-0002-2494-3279. Universidad del País Vasco, España. mariateresa.santos@ehu.eus

2 orcid.org/0000-0002-3383-4859. Universidad del País Vasco, España. jesusangel.perez@ehu.eus 
que general. No obstante, su presencia se incrementa los meses de diciembre, febrero y marzo $(64,3 \%)$, fechas que coinciden con celebraciones o la publicación de noticias tratadas con cierto sensacionalismo que crean una gran atención mediática. Asimismo, se constata la necesidad de la especialización de los profesionales y el uso de las fuentes expertas.

\section{Palabras clave (fuente: Tesauro de la Unesco)}

Enfermedades raras; salud; medios de comunicación; investigación; España. 


\section{Rare Diseases and their Representation in the Spanish Press}

\section{Abstract}

This article derives from a research project focused on the media attention given by Spanish newspapers El País, ABC, El Mundo and La Razón to rare diseases (RDs) between October 1, 2016 and September 30, 2017. This is a live issue of social interest that contributes to spotlighting a serious health problem suffered by less than five out of 10,000 people. These pathologies are chronic, life-threatening and highly likely to cause disability and dependency. By means of a content analysis method, thematic areas are identified, and the treatment given to them by such newspapers is quantified, analyzed and compared. Results show that it is a relevant emerging issue in the national press, which is dealt with in generic texts that are mostly posted in the society section and prepared with little depth and hardly any explanations or opinions using a single source. The most recurrent themes are medicine/diseases, research and courts. Therefore, $\mathrm{RDs}$ are present in Spanish newspapers that convey a view to society that is comparable to other diseases and provide a general approach. However, their presence increases in December, February and March (64.3\%), which coincide with celebrations or the publication of sensationalist news that attracts great media attention. The need for specialized professionals and expert sources is also confirmed.

\section{Keywords (Source: Unesco Thesaurus)}

Strange diseases; health; mass media communications; research; Spain. 


\section{As doenças raras e sua representação na imprensa espanhola}

\section{Resumo}

Este artigo é o resultado de uma pesquisa focada na atenção midiática que os jornais espanhóis El País, ABC, El Mundo e La Razón dão às doenças raras desde $1^{\circ}$ de outubro de 2016 até 30 de setembro de 2017. Um tema de atualidade e interesse social que contribui para dar visibilidade a um grave problema de saúde que menos de cinco pessoas de cada dez mil padecem. Essas patologias são caracterizadas por serem crônicas, potencialmente mortais e com alto grau de deficiência e dependência. Utilizando uma metodologia de análise de conteúdo, são identificadas as áreas temáticas, e é quantificado, analisado e comparado o tratamento realizado pelos jornais mencionados. Os resultados mostram que é um tema emergente e relevante na imprensa nacional. Trata-se de textos genéricos que são incluídos, na maioria dos casos, na seção de sociedade e elaborados com escasso aprofundamento, sem explicações nem opiniões, tendo em vista que somente utilizam uma fonte. As temáticas mais recorrentes são medicina ou doenças, pesquisa e tribunais. Portanto, as doenças raras estão presentes nos jornais espanhóis e transmitem à sociedade uma visão que se compara às demais doenças, contribuindo com uma abordagem geral. Contudo, sua presença é aumentada nos meses de dezembro, fevereiro e março $(64,3$ $\%)$, datas que coincidem com celebrações ou publicação de notícias tratadas com certo sensacionalismo, que criam uma grande atenção midiática. Além disso, é constatada a necessidade da especialização dos profissionais e do uso de fontes especializadas.

\section{Palavras-chave (Fonte: Tesauro da Unesco)}

Doenças raras; saúde; meios de comunicação; investigação; Espanha. 


\section{Introducción}

Las enfermedades raras (ER) se caracterizan por tener una prevalencia baja, ser crónicas y potencialmente mortales, además presentan un alto grado de discapacidad y dependencia y con ello un deterioro significativo en su calidad de vida (Avellaneda, Izquierdo, Torrent-Farnell y Ramón, 2017). Es decir, la padecen menos de cinco personas por cada 10 000, según estimación del Programa de Acción Comunitaria sobre las enfermedades poco comunes (1999-2003), aprobado por el Consejo y el Parlamento Europeo (Decisión no 1295/1999/CE del Parlamento Europeo y del Consejo, de 29 de abril de 1999, por la que se aprueba un programa de acción comunitaria sobre las enfermedades poco comunes en el marco de la acción en el ámbito de la salud pública (1999-2003)). No obstante, no existe un criterio unificado, sino que depende del país. Así, en los Estados Unidos, se califica como tal si afecta a menos de 200000 personas, según la Orphan Drug Act de 1983, que sirvió para fomentar la investigación y el desarrollo de fármacos para las ER y sus posibles curas. Por el contrario, en Japón, desciende a 50000 personas (tasa de 3,9\%) y en Australia a menos de 2000 personas (tasa de 0,9\%) (Torrent-Farnell y Morros, 2001).

Se trata de enfermedades con unos síntomas que comienzan a manifestarse antes de los dos años y presentan un elevado grado de discapacidad y dependencia, cronicidad, complejidad etiológica y diagnóstica, y que son potencialmente mortales (Lugones y Ramírez, 2012). Además, su diagnóstico con frecuencia supone años, tiempo en el cual se incrementa su problemática física, psicológica y social (García, Verde, Suerio, Rosado y Sorlí, 2013). Esta situación supone un cambio importante en sus vidas por las secuelas que ocasionan (Huerta, Fernández, Gallardo y Hernández, 2012). No se puede olvidar que influye en los aspectos físicos, biológicos, laborales, emocionales, sociales y familiares y, en consecuencia, surgen dificultades para su integración normal en los procesos sociales habituales (Salinas, Rogero, Oña y Vergara, 2012, citados por Seco y RuizCallado, 2016). También constituye un reto científico para lograr el mayor grado de calidad de vida para los afectados (Schieppati, Henter, Daina y Aperia, 2008; Chaves, s. f.). 
Son numerosos los estudios sobre el tema realizados en el ámbito sanitario de la comunicación (Sontang, 1978; Yardley,1997). Más recientemente han analizado la salud en los medios otros autores (Blanco y Paniagua, 2007; Calvo, 2002; Carrillo, 2004; Cavaca, Emerich, VasconcellosSilva, Dos Santos-Neto y Oliveira, 2016; Cavaca, Vasconcellos-Silva, Ferreira y Nunes, 2015; Costa, 2008; Dalley, Buunk y Umit, 2009; Emerich, Cavaca, Santos-Neto, Gentilli y Oliveira, 2017; Jacobs, Boyd, Brennan, Sinha y Giuliani, 2016; Fernández y Pritchard, 2012; González, 2004; Revuelta, 2006; Revuelta, De Semir y Minelli, 1997; Schumaker et al., 2014). A su vez, otros autores tratan aspectos más concretos de las enfermedades, por ejemplo, la gripe A (Camacho, 2009), el VIH/sida (Igartua y Lopes, 2002; Terrón, 2012), la obesidad (Westall, 2011; Yoo y Kim, 2012), el ébola (Adekunle y Adnan, 2016), la salud mental (Muñoz, Pérez-Santos, Crespo, Guillén y Izquierdo, 2011; Peñafiel, Camacho, Aiestaran, Ronco y Echegaray-Eizaguirre, 2014), la anorexia (Guzmán y Rodríguez, 2016), la discapacidad intelectual (Rodríguez y Ferreira, 2010), los fármacos (Huertas y Xifra, 2009), periodismo biomédico (Moreno, 2010) y la salud en prensa gratuita (Santos, 2008, 2009). No obstante, es un campo de investigación nuevo, dado que hasta 2007 no se sientan los fundamentos teóricos para el análisis del discurso periodístico, aunque referido a discapacidad y comunicación (Bañón, 2007; Bañón y Requena, 2014; Bañón et al., 2011; Palau, 2010), le siguen estudios que tratan la discapacidad en general (Arcos, 2013; Rius y Solves, 2009; Sánchez-Hernández, 2016; Solves y Bañón, 2014). Otros investigadores se centran en patologías concretas como el síndrome de Asperger (Rodríguez, 2007) o el de Guilles de la Tourette (Férriz, 2008); sobre psicología, salud y red (Arcos 2013; Fornieles y Bañón, 2011); acerca de la prevalencia (Palau, 2010); el enfoque social de la enfermedad (Seco y Ruiz-Callado, 2016). Por otra parte, algunos autores demuestran el interés por las ER en prensa mediante la teoría del framing y concluyen cómo las ER buscan visibilidad a través de los medios junto con recursos económicos (Bañón et al., 2011; Díaz, 2007; Fornieles y Bañón, 2011; Navarro, 2004; Rius y Solves, 2006; Sánchez-Castillo, 2012, 2013; Sánchez-Castillo y Mercado, 2014), que destaca la necesidad de desarrollar políticas de comunicación eficientes, trabajos que han servido de base a nuestra investigación. Por su parte, los resultados de López-Villafranca 
(2016) y López-Villafranca y Castillo-Esparcia (2018) muestran que los medios publican las noticias con contenidos de agencia y por tanto con escasa elaboración. Otros aspectos de interés son la comunicación y sociedad (Aymé y Schmidtke, 2007), y comunicación de pacientes y gabinetes de comunicación (López-Villafranca, 2016). Como se puede observar, se han abordado las ER desde el punto de vista sanitario, de la psicología, de la discapacidad, mientras que las referidas a prensa española se centran en el análisis de los encuadres noticiosos. No se puede olvidar que es un tema emergente para los profesionales de los medios tanto especializados como generalistas, y con cierta complejidad en el uso de la terminología específica (Bañón y Requena, 2014). A pesar de la trascendencia social, son escasas las investigaciones realizadas sobre su tratamiento en los medios de comunicación desde el ámbito académico en general y desde el área de la comunicación en particular (Bañón, 2007; Sánchez Castillo y Mercado, 2014). Este estudio muestra el reflejo de las ER en los medios de comunicación españoles, prestando también atención a la manera en que el periodista aborda los contenidos (géneros, autoría, fuentes y secciones). Por tanto, se trata de un tema de actualidad e interés social que contribuye a dar visibilidad a un grave problema de salud aportando una visión general y diferente del tratamiento otorgado en los diarios de información general españoles sobre las ER en El País, El Mundo, La Razón y ABC, entre el 1 de octubre de 2016 y el 30 de septiembre de 2017.

\section{Percepción social de las enfermedades raras}

Los medios de comunicación desempeñan un papel determinante a la hora de dar visibilidad a las ER (Adekunle y Adnan, 2016; Cavaca et al., 2016), y más cuando se trata de afectados y familiares acompañados por representantes institucionales (Sánchez-Castillo, 2012). Necesitan buscar una visibilidad con el fin de mostrar a la sociedad las consecuencias sanitarias, sociales y económicas que provocan estas patologías (LópezVillafranca, 2016). En este contexto, en 2012, inician su andadura las organizaciones de pacientes, que junto con las asociaciones proporcionan apoyo emocional y terapéutico al compartir experiencias comunes (Huerta, Fernández, Gallardo y Hernández, 2012). No obstante, cuentan con escasos recursos y pocos socios, por lo que se ven en la necesidad de 
constituirse jurídicamente bajo esta forma para captar fondos (Mosquera, 2002). En el ámbito internacional, son referencia para Europa la European Organisation for Rare Diseases (Eurordis) y para América Latina la Alianza Iberoamericana de Enfermedades Raras o Poco Frecuentes (Aliber). En el territorio nacional, en 1999, surge la Federación Española de Enfermedades Raras (Feder). En conjunto, todas ellas centran sus objetivos en coordinar pacientes, Administración pública, científicos, profesionales de la salud e industria para conseguir mejorar la calidad de la atención a los enfermos.

Para dar visibilidad a las ER, el 28 de febrero, se celebra en Europa, los Estados Unidos, América Latina, Canadá y Asia el Día Mundial de las Enfermedades Raras con la realización de numerosas actividades que cuentan con el apoyo de personajes relevantes. Un ejemplo lo tenemos en la exposición Expression of Hope inaugurada por la reina Letizia; El Telemaratón emitido por TVE con motivo de la celebración de su día internacional con el objetivo de recaudar fondos para la investigación, en el que participan periodistas como Isabel Gemio, J. Vázquez, Aberasturi, J. Cárdenas; futbolistas como Ronaldo; cantantes como Manu Tenorio, Alejandro Sanz; actores como Dani Rovira, Eleven, Wisin; el torero Ortega Cano; el científico Matesanz, entre otros. La presencia de personalidades da relevancia al evento, pero se debe evitar que su notoriedad eclipse o incluso anule a los afectados (Arcos, 2013; Bañón et al., 2011).

Los colectivos relacionados con las ER también publican revistas especializadas. Entre ellas, Genoma, Investigación y Sociedad, que nació con el objetivo de convertirse en punto de encuentro multidisplinar entre pacientes, asociaciones, laboratorios, personal sanitario especializado y Administración. Orphanet Journal of Rare Diseases y Rare Diseases abordan, además de la enfermedad, los medicamentos huérfanos; The Journal of Rare Disorders, que surgió en los Estados Unidos con el objetivo de proporcionar un foro para los médicos, profesionales de la salud, defensores e investigadores. En España, newsRARE centrada en la divulgación científica. En conjunto, se trata de revistas especializadas destinadas a la difusión, el debate y el intercambio de experiencias y opiniones de este tipo de patologías. 
Dentro de su labor divulgadora, se encuentran los libros para niños. Sus contenidos van desde relatos sobre la integración hasta la sostenibilidad, como Cuentos para ser escuchados, Diálogos con Axel, Cuando seamos inmortales, El cazo de Lorenzo; en otros casos, tratan historias de superación, como El beso de la estrella escrito por una persona afectada por nevus congénito gigante; las experiencias sobre discapacidad intelectual son contadas en Bienvenidos a Holanda (Wellcome to Holland) o Mi hermana Lola. También existen libros solidarios en los que el autor cede parte de sus beneficios, como La magia de la leche, El libro rojo de la logística o Te quiero mucho, entre otros.

Los entornos digitales facilitan el acceso a la información sobre avances científicos y posibilitan el intercambio de experiencias y consejos sobre diversas patologías. Con esta finalidad, surge en Europa RareConnect una red social dirigida por pacientes de comunidades de ER promovida por la Eurordis y la National Organization for Rare Disorders (NORD) y Orphanet. Tareas que se ven complementadas con las asociaciones de ER, que aprovechan las posibilidades que ofrece la tecnología para realizar labores de difusión, participación, comunicación, coordinación, organización, apoyo y divulgación científica. En este sentido, las redes sociales como Twitter y Facebook se han convertido en una nueva herramienta para dar visibilidad, además de integración y comunicación capaz de responder a las necesidades de este colectivo (Santos y Pérez, 2017).

En algunas ocasiones, las ER resultan tan extrañas que se han llevado al cine para llamar la atención dado el impacto que producen (García y García, 2005). Algunos ejemplos son Lorenzo's Oil (1992), de George Miller, que cuenta la búsqueda de la curación de unos padres para su hijo que padece adrenoleucodistrofia. Otros filmes tratan síndromes como el de Morquio en Simon Birch (1998), de Mark Steven Johnson; de Tourette en The Tic Code (1999), de Gary Winick; de Asperger en Mary and Max (2009). A su vez, Adam Elliot presenta Mozart and the Whale (2005); el director Max Mayer presenta Adam (2009) y Ben X (2007) dirigida por Nik Balthasar. Otra enfermedad como es la agnosia está presente en filmes como La agnosia (2010), dirigida por Eugenio Mira; y Más allá del espejo (2006), de Joaquim 
Jordà. Otras películas relacionadas con las ER son Extraordinary Measures (2010), de Tom Vaughan; la acondroplasia se refleja en The Station Agent (2003), dirigida por Thomas McCarthy. La enfermedad de los huesos de cristal se muestra en El secreto de Amila (2015), dirigida por Gorka Vázquez; y Alejandro Amenábar aborda la xeroderma pigmentoso en Los otros (2001). En definitiva, se trata de iniciativas encaminadas a dar visibilidad a estas enfermedades, fomentar la investigación, ayudar en la planificación sanitaria y en una correcta distribución de recursos.

\section{Objetivos e hipótesis}

Este estudio forma parte de una investigación más amplia que tiene como objetivo el análisis del tratamiento que realiza la prensa española de información general sobre las ER. Con el fin de conseguir nuestro propósito, se parte de los siguientes objetivos:

- Cuantificar las informaciones publicadas en cada diario mencionado sobre ER.

- Determinar su cobertura informativa.

- Identificar las áreas temáticas que abordan los diarios.

- Comparar el tratamiento informativo que hace cada diario de las ER.

Con el fin de conseguir los objetivos planteados, partimos de los siguientes interrogantes:

- ¿ ¿Las ER forman parte de la agenda de los medios?

- ¿Los textos sobre ER son de carácter eminentemente informativo? ¿Con qué grado de profundidad están elaborados?

- ¿Qué temáticas predominan en los contenidos sobre ER publicados por los diarios analizados?

\section{Metodología}

Para realizar el estudio, se han seleccionado las cabeceras de los diarios El País (1976), El Mundo (1989), ABC (1903) y La Razón (1998). En la elección de la muestra, se han tenido en cuenta criterios como la 
calidad de los periódicos, su cobertura nacional, amplia difusión y líneas editoriales diferentes que cubren todos los espacios sociológicos. También su pertenencia a grupos de comunicación consolidados: Prisa (El País), Unidad Editorial S. A. (El Mundo), Vocento $(A B C)$ y Planeta (La Razón). Por tanto, son periódicos de referencia, creadores de opinión y que resultan adecuados para el estudio. Como espacio temporal, se ha elegido desde 1 de octubre de 2016 hasta el 30 de septiembre de 2017. Como unidad de análisis se contempla cada una de las piezas publicada en los diarios, con independencia del género periodístico empleado (informativo, interpretativo o de opinión), siguiendo criterios establecidos en otros estudios (Camacho, 2009; Santos Diez y Pérez, 2017), que contenga el término "enfermedad rara", una acepción aceptada mayoritariamente por distintos autores (Arcos, 2013; Sánchez-Hernández, 2016; Sánchez y Mercado 2014; Solves y Bañón, 2014; Rius y Solves, 2009). La búsqueda de las noticias se ha realizado desde los portales web de los diarios mencionados y se han seleccionado todas las piezas que tenían como tema principal estas enfermedades, descartándose aquellas que lo tratan de manera circunstancial. Como resultado de la revisión, se han contabilizado 304 unidades de análisis $A B C(\mathrm{n}=83,27,3 \%)$, El País $(\mathrm{n}=94,30,9 \%), E l$ Mundo $(\mathrm{n}=66,21,8 \%)$ y La Razón $(\mathrm{n}=61,20 \%)$.

Para analizar los textos, se procedió al diseño de una ficha específica con el fin de poder codificar los datos con las siguientes variables según las pautas de Solves y Bañón (2014):

- Fecha de publicación.

- $\quad$ Autoría: redacción, agencia, sin firma, redactor, otros.

- Género periodístico: noticia, reportaje, entrevista, opinión y otros.

- Imágenes: con fotografía, sin fotografía, otros.

- Secciones: salud, sociedad, ciencia, nacional, opinión, sucesos, comunicación, sanidad, economía, familia y otros.

- Fuentes: juzgado, policiales, científicas, hospitales, universidad, institucionales, periódicos, familia, asociaciones, entrevistados, sin identificar y otras. 
- Temática: investigación, recursos, solidaridad, fallecimientos, tribunales, enfermedades, asociaciones, medicina, eventos y otros.

Para probar la fiabilidad del desarrollo de la codificación (intercoder reliability), se tomaron al azar 60 piezas (el $20 \%$ del total), que fueron cifradas en cada una de las variables utilizadas por dos investigadores de manera independiente, obteniendo unos resultados finales de 57 y 56 coincidencias en las mediciones totales. El coeficiente Kappa de Cohen (K) nos dio una fiabilidad del 0,88 \% y un índice de confianza del 94,1\%, de modo que quedan garantizados los resultados obtenidos (Landis y Koch, 1977). Para lograr los objetivos propuestos, se ha utilizado una metodología basada en el análisis de contenido. Una técnica propuesta por autores de referencia en este campo como Bardin (2002), Berelson (1952), Igatua (2006), Holsti (1969), Krippendorf (1980) y Piñuel y Gaitán (1995). Además, permite el estudio de los textos publicados de una forma sistemática, objetiva y cuantitativa (Wimmer y Dominick, 1996), y ofrece al investigador la fecundidad de la subjetividad para encontrar lo oculto, lo no aparente encerrado en todo mensaje (Bardin, 2002).

\section{Resultados}

Durante el periodo analizado en los cuatro diarios, se han contabilizado 304 textos sobre las ER distribuidas del siguiente modo: El País 94 (30,9\%), ABC 83 (27,3\%), El Mundo 66 (21,8\%) y La Razón 61 (20\%).

\section{Presencia de las ER en la agenda de los medios}

Según la tabla 1, los meses de diciembre $(29,4 \%)$, febrero $(19,4 \%)$ y marzo $(15,5 \%)$ publican más noticias sobre ER, mientras que en junio (2\%) desciende el interés. No obstante, se producen discrepancias entre los diarios: así El País presta más atención en los meses de diciembre $(36,2 \%)$ y marzo (13,9\%); $A B C$ lo hace en febrero $(33,7 \%)$ y diciembre (27,7\%); La Razón en diciembre (29,7\%) y febrero (18\%); y El Mundo en diciembre (21,2\%) y marzo (19,7\%). Algo lógico si se tiene en cuenta que en diciembre se produce el caso Nadia, en febrero se celebra el Día Mundial de las Enfermedades Raras y en marzo el caso de los 2000 tumores. 


\section{Tabla 1. Distribución de los textos por mes (\%)}

\begin{tabular}{|l|c|c|c|c|c|}
\hline \multicolumn{1}{|c|}{ Mes } & $\begin{array}{c}\text { La Razón } \\
(\mathbf{n = 6 1 , 2 0} \%)\end{array}$ & $\begin{array}{c}\text { El Mundo } \\
(\mathbf{n = 6 6 , 2 1 , 8} \%)\end{array}$ & $\begin{array}{c}\text { El País } \\
(\mathbf{n}=\mathbf{9 4 , 3 0 , 9} \text { \% })\end{array}$ & $\begin{array}{c}\text { ABC } \\
(\mathbf{n}=\mathbf{8 3}, \mathbf{2 7}, \mathbf{3} \%),\end{array}$ & $\begin{array}{c}\text { Total textos } \\
(\mathbf{3 0 4})\end{array}$ \\
\hline Octubre & 3,3 & 10,6 & 3,2 & 3,7 & $15(5 \%)$ \\
\hline Noviembre & 4,9 & 3 & 4,2 & 3,7 & $12(4 \%)$ \\
\hline Diciembre & 29,7 & 21,2 & 36,2 & 27,7 & $89(29,4 \%)$ \\
\hline Enero & 3,3 & 7,6 & 11,9 & 9,6 & $26(8,5 \%)$ \\
\hline Febrero & 18 & 13,6 & 11,9 & 33,7 & $59(19,4 \%)$ \\
\hline Marzo & 13,1 & 19,7 & 13,9 & 15,6 & $47(15,5 \%)$ \\
\hline Abril & 4,9 & 4,6 & 1 & 1,2 & $8(2,6 \%)$ \\
\hline Mayo & 3,3 & 7,6 & 6,4 & 0 & $13(2,3 \%)$ \\
\hline Junio & 1,6 & 3 & 2 & 1,2 & $6(2 \%)$ \\
\hline Julio & 4,1 & 3 & 4,1 & 1,2 & $12(4 \%)$ \\
\hline Agosto & 4,9 & 1,5 & 4,1 & 1,2 & $9(3 \%)$ \\
\hline Septiembre & 4,6 & 1,2 & $8(2,6 \%)$ \\
\hline
\end{tabular}

Fuente: elaboración propia.

Con respecto a la procedencia de la información, predomina el ámbito estatal $(89,4 \%)$ frente al internacional $(10,6 \%)$, aunque existen diferencias entre los periódicos: La Razón (95,1 \%), El Mundo (92,4 \%), ABC (91,6) y El País (80,9 \%). Como se muestra en la tabla 2, en el Estado español, las comunidades autónomas que más cobertura informativa reciben son Madrid (48\%), aunque con discrepancias entre los diarios. Destaca con el 67,2 \% en La Razón, el 50,6 \% en ABC, un 40,4 \% en El País y El Mundo el $38 \%$. Le sigue Cataluña (19\% ), con el $34 \%$ en El País, el 14,4\% en ABC, el 13,6 \% en El Mundo y en $L$ a Razón el 8,3 \%. Existe un interés local y regional que destaca la proximidad como valor de la noticia que adquiere relevancia inmediata. En el ámbito internacional, los países que acaparan mayor interés informativo son el Reino Unido (2,7 \%) seguido de los Estados Unidos $(2,4 \%)$ y la India $(1,6 \%)$. 


\section{Tabla 2. Lugar de procedencia geográfica de la información (\%)}

\begin{tabular}{|c|c|c|c|c|c|}
\hline Lugar & $\begin{array}{c}\text { La Razón } \\
(n=61,20 \%)\end{array}$ & $\begin{array}{c}\text { El Mundo } \\
(\mathrm{n}=66,21,8 \%)\end{array}$ & $\begin{array}{c}\text { El País } \\
(n=94,30,9 \%)\end{array}$ & $\begin{array}{c}\mathrm{ABC} \\
(\mathrm{n}=\mathbf{8 3}, \mathbf{2 7 , 3} \%)\end{array}$ & $\begin{array}{c}\text { Total textos } \\
\quad 304\end{array}$ \\
\hline Madrid & 67,2 & 38 & 40,4 & 50,6 & $146(48 \%)$ \\
\hline Cataluña & 8,3 & 13,6 & 34 & 14,4 & $58(19 \%)$ \\
\hline Comunidad Valenciana & 4,9 & 15,2 & 5,3 & 9,5 & $26(8,6 \%)$ \\
\hline Castilla-León & 9,8 & 0 & 0 & 4,8 & $10(3,3 \%)$ \\
\hline Castilla-La Mancha & 0 & 1,5 & 0 & 9,5 & $9(3 \%)$ \\
\hline Andalucía & 3,3 & 4,6 & 0 & 1,2 & $6(1,8 \%)$ \\
\hline Islas Baleares & 0 & 7,6 & 0 & 1,2 & $6(1,8 \%)$ \\
\hline Alicante & 0 & 6 & 0 & 0 & $4(1,2 \%)$ \\
\hline Canarias & 1,6 & 3 & 0 & 0 & $3(0,9 \%)$ \\
\hline Valencia & 0 & 1,5 & 11,1 & 1,2 & $3(0,9 \%)$ \\
\hline País Vasco & 0 & 1,5 & 0 & 0 & $1(0,3 \%)$ \\
\hline Total España & 95,1 & 92,4 & 80,9 & 91,6 & 272 \\
\hline Reino Unido & 1,6 & 3 & 5,3 & 0 & $8(2,7 \%)$ \\
\hline Estados Unidos & 3,3 & 11,5 & 3,3 & 1,2 & $7(2,4 \%)$ \\
\hline India & 0 & 1,5 & 2 & 2,4 & $5(1,6 \%)$ \\
\hline Vaticano & 0 & 0 & 0 & 2,4 & $2(0,6 \%)$ \\
\hline Corea & 0 & 0 & 2 & 0 & $2(0,6 \%)$ \\
\hline México & 0 & 0 & 1 & 0 & $1(0,3 \%)$ \\
\hline China & 0 & 0 & 1,1 & 0 & $1(0,3 \%)$ \\
\hline Francia & 0 & 0 & 1,1 & 0 & $1(0,3 \%)$ \\
\hline Columbia & 0 & 0 & 1,1 & 0 & $1(0,3 \%)$ \\
\hline Unión Europea & 0 & 1,5 & 0 & 0 & $1(0,3 \%)$ \\
\hline Suiza & 0 & 0 & 1,1 & 0 & $1(0,3 \%)$ \\
\hline Sierra Leona & 0 & 0 & 11,1 & 0 & $1(0,3 \%)$ \\
\hline Nigeria & 0 & 0 & 0 & 1,2 & $1(0,3 \%)$ \\
\hline Total internacional & 4,9 & 7,6 & 19,1 & 8,4 & 32 \\
\hline
\end{tabular}

Fuente: elaboración propia. 


\section{Géneros, autoría, sección y fuentes}

En cuanto se refiere a los géneros periodísticos empleados (tabla 3), hay un claro predominio de la noticia $(83,7 \%)$ en todos los diarios ( $E l$ Mundo 89,5 \%, La Razón 83,6 \%, ABC 81,9 \% y El País 80,9 \%). En menor medida, está el reportaje ( $E l$ País 10,6 \% y $A B C 7,3 \%$ ) y la entrevista ( $L a$ Razón 8,3 \% y ABC 5,3\%). Los resultados muestran un predominio de los géneros informativos, $y$ destaca la escasa presencia del reportaje $(5,9 \%)$ y la entrevista $(5,9 \%)$ frente a la opinión y los interpretativos.

Tabla 3. Género empleado (\%)

\begin{tabular}{|l|c|c|c|c|c|}
\hline \multicolumn{1}{|c|}{ Género } & $\begin{array}{c}\text { La Razón } \\
(\mathbf{n = 6 1 , 2 0} \%)\end{array}$ & $\begin{array}{c}\text { El Mundo } \\
(\mathbf{n}=\mathbf{6 6}, \mathbf{2 1}, \mathbf{8} \%)\end{array}$ & $\begin{array}{c}\text { El País } \\
(\mathbf{n}=\mathbf{9 4 , 3 0 , 9} \%)\end{array}$ & $\begin{array}{c}\mathbf{A B C} \\
(\mathbf{n}=\mathbf{8 3}, \mathbf{2 7}, \mathbf{3} \%)\end{array}$ & $\begin{array}{c}\text { Total textos } \\
(\mathbf{3 0 4})\end{array}$ \\
\hline Noticia & 83,6 & 89,5 & 80,9 & 81,9 & $254(83,7 \%)$ \\
\hline Reportaje & 1,6 & 1,5 & 10,6 & 7,3 & $18(5,9 \%)$ \\
\hline Entrevista & 8,3 & 6 & 2,1 & 5,3 & $18(5,9 \%)$ \\
\hline Opinión & 4,9 & 1,5 & 6,4 & 5,5 & $12(3,9 \%)$ \\
\hline Otros & 1,6 & 1,5 & 0 & 0 & $2(0,6 \%)$ \\
\hline
\end{tabular}

Fuente: elaboración propia.

En cuanto a la autoría de los textos, es significativo el alto porcentaje de los que se publican con firma del redactor $(71,7 \%)$, aunque en los diarios La Razón (88,7 \%) y El País $(85,2 \%)$ hay una proporción más elevada que en $E l$ Mundo (71,3 \%) y $A B C(44,5 \%)$. Le siguen los de agencia con el $11,8 \%$ del total, distribuidos del siguiente modo: $A B C 28,9 \%$, El Mundo 12,1 \%, El País 3,2 \% y La Razón 1,6 \%. Resultan relevantes el número de informaciones publicadas sin firma en La Razón (8,1 \%), ABC (3,7\%), El Mundo (2,3\%) y El País (1\%), y con nombre del periódico (11,8\%), aunque con diferencias entre los diarios ( $A B C 22,9 \%$, El Mundo 12,1 \%, El País 8,5\% y La Razón 1,6\%). Se trata, en general, de textos que se publican prácticamente sin ninguna intervención por parte de los periodistas que las escriben (Gelado-Marcos, 2009; Bañón y Requena, 2014). 
Por otro lado, los diarios analizados sitúan un mayor número de noticias sobre ER en la sección de sociedad con el 33,5 \%, distribuidas del siguiente modo: ABC 53 \%, La Razón 47,5 \%, El Mundo 31,9 \% y El País $8,5 \%$. A continuación va la sección nacional con el $24,3 \%$ repartida de la siguiente manera: El País 58,5 \%, ABC 13,3 \% y El Mundo 12,2\%. Asimismo, cabe destacar secciones como salud (16,8\%) con porcentajes inferiores y un reparto desigual (La Razón 29,5 \%, El Mundo 28,8 \%, El País 10,7\% y $A B C 4,8 \%$ ).

En los diarios analizados, el 94,7 \% tienen acompañamiento gráfico, en su mayoría se trata de fotografías de personas relacionadas con la familia $(59,5 \%)$ o el propio afectado (28,9\%). Porcentajes que suponen el 94,8\% en El País, el 86,4 \% en El Mundo, el 86,8 \% en ABC y La Razón el 83,6 \%. Son fotografías informativas, dado que no aportan al texto elementos complementarios, además se repiten en distintos contenidos y periódicos (Camacho, 2009). En cierto modo, se puede considerar que "va dirigida a lo emocional, nos persuade con mayor eficacia y nos llega de modo global sin que muchas veces seamos conscientes de las inferencias y los detalles que captamos inconscientemente" (Fornieles y Bañón, 2011, p. 189). También cabe mencionar que en el 6,2 \% se incluyen otros recursos gráficos (El País 4,2 \%, El Mundo 6 \%, La Razón 8,2 \% y ABC 7,2\%).

Como se observa en la tabla 4, las fuentes científicas son las más numerosas con un 22,5 \% del total (La Razón 32,9 \%, El País, 28,7 \%, El Mundo, 21,3\% y ABC, 8,4\%). Destaca la accesibilidad de fuentes relevantes y expertas que pueden contribuir a que ciertas enfermedades sean más visibles y tengan mayor cobertura en los medios (Cavaca et al., 2016). Le siguen a distancia la familia $(13,2 \%)$, aunque con diferencias entre los diarios (ABC, 22,9 \%, El País 13,9 \%, El Mundo, 7,6 \% y La Razón 4,9 \%), e institucionales con un 9,8 \% (El Mundo, 19,7 \%, ABC, 10,8 \%, La Razón 8,3 \% y El País, 3,2 \%). Asimismo, se comprobó que la información está escasamente contrastada, dado que se publica utilizando una única fuente en 218 piezas, es decir, el 71,7 \% de los textos (La Razón 83,8 \%, ABC 77,2 \%, El Mundo 72,9 \% y El País 58,6 \%). Le siguen a mucha distancia los textos $(13,8 \%)$ que utilizan dos fuentes (El País 20,2 \%, El Mundo 13,6 \%, ABC 
10,8 \% y La Razón 8,2 \%). A su vez, destaca el escaso porcentaje del uso de cuatro fuentes con un 5,6 \% (El País 10,6 \%, ABC 4,8 \%, La Razón 3,2 \% y El Mundo 1\%) (tabla 4).

Tabla 4. Fuentes utilizadas en los diarios (\%)

\begin{tabular}{|c|c|c|c|c|c|}
\hline & $\begin{array}{c}\text { La Razón } \\
(\mathrm{n}=\mathbf{6 1}, \mathbf{2 0} \%)\end{array}$ & $\begin{array}{c}\text { El Mundo } \\
(\mathrm{n}=66,21,8 \%)\end{array}$ & $\begin{array}{c}\text { El País } \\
(n=94,30,9 \%)\end{array}$ & $\begin{array}{c}\mathrm{ABC} \\
(\mathrm{n}=\mathbf{8 3}, \mathbf{2 7 , 3} \%)\end{array}$ & $\begin{array}{c}\text { Total de textos } \\
(304)\end{array}$ \\
\hline Científicas & 32,9 & 21,3 & 28,7 & 8,4 & $68(22,5 \%)$ \\
\hline Familia & 4,9 & 7,6 & 13,9 & 22,9 & $40(13,2 \%)$ \\
\hline Juzgado & 9,9 & 4,6 & 19,4 & 4,8 & $31(10,2 \%)$ \\
\hline Institucional & 8,3 & 19,7 & 3,2 & 10,8 & $30(9,9 \%)$ \\
\hline Asociación & 8,3 & 6 & 7,4 & 16,9 & $30(9,9 \%)$ \\
\hline Hospitales & 4,9 & 13,6 & 5,3 & 12,2 & $27(8,9 \%)$ \\
\hline Sin identificar & 16,4 & 9 & 5,3 & 4,8 & $25(8,2 \%)$ \\
\hline Universidad & 3,2 & 6 & 4,2 & 2,4 & $12(3,9 \%)$ \\
\hline Entrevistado & 1,6 & 3 & 4,2 & 6 & $12(3,9 \%)$ \\
\hline Periódico & 3,2 & 4,6 & 3,2 & 3,6 & $11(3,6 \%)$ \\
\hline Policiales & 3,2 & 0 & 4,2 & 3,6 & $9(2,9 \%)$ \\
\hline Otros & 3,2 & 4,6 & 1 & 3,6 & $9(2,9 \%)$ \\
\hline
\end{tabular}

Fuente: elaboración propia.

\section{Temáticas}

En cuanto a los temas más destacados, como recoge la tabla 5 , son medicina/enfermedades $(34,6 \%)$, investigación (22\%) y tribunales $(13,6 \%)$, y en menor medida aparecen solidaridad $(9,5 \%)$ y asociaciones (5,9\%). Coinciden en dar mayor relevancia a los contenidos de medicina/ enfermedades (La Razón 29,5 \%, El País 31,9 \% y ABC 47 \%), mientras que $E l$ Mundo da prioridad a la investigación (33,5\%). Otros contenidos de interés son para La Razón la investigación (26,3\%) y recursos (16,4\%); en $E l$ Mundo destaca medicina/enfermedades $(27,4 \%)$ y asociaciones (10,6 \%). A su vez, El País resalta tribunales (29,8 \%) e investigación $(19,3 \%)$, mientras que $A B C$ centra la atención en solidaridad (18\%) e investigación $(13,3 \%)$. 


\section{Tabla 5. Temática}

\begin{tabular}{|l|c|c|c|c|c|}
\hline & $\begin{array}{c}\text { La Razón } \\
(\mathbf{n}=\mathbf{6 1}, \mathbf{2 0} \%)\end{array}$ & $\begin{array}{c}\text { El Mundo } \\
(\mathbf{n}=\mathbf{6 6}, \mathbf{2 1} \mathbf{8})\end{array}$ & $\begin{array}{c}\text { El País } \\
(\mathbf{n}=\mathbf{9 4 , 3 0 , 9} \text { \%) }\end{array}$ & $\begin{array}{c}\mathbf{A B C} \\
(\mathbf{n = 8 3}, \mathbf{2 7}, \mathbf{3} \%)\end{array}$ & $\begin{array}{c}\text { Total textos } \\
(\mathbf{3 0 4})\end{array}$ \\
\hline Medicina/enfermedades & 29,5 & 27,4 & 31,9 & 47 & $105(34,6 \%)$ \\
\hline Investigación & 26,3 & 33,5 & 19,3 & 13,3 & $67(22 \%)$ \\
\hline Tribunales & 1,6 & 9 & 29,8 & 7,3 & $41(13,6 \%)$ \\
\hline Solidaridad & 11,5 & 9 & 1 & 18 & $29(9,5 \%)$ \\
\hline Recursos & 16,4 & 4,5 & 4,2 & 4,8 & $21(6,9 \%)$ \\
\hline Asociaciones & 4,9 & 10,6 & 5,3 & 3,6 & $18(5,9 \%)$ \\
\hline Otros & 4,5 & 4,5 & 5,3 & 2,4 & $13(4,3 \%)$ \\
\hline Fallecimientos & 1,6 & 0 & 3,2 & 2,4 & $6(1,9 \%)$ \\
\hline Eventos & 3,3 & 1,5 & 0 & 1,2 & $4(1,3 \%)$ \\
\hline
\end{tabular}

Fuente: elaboración propia.

\section{Medicina/enfermedades}

Este apartado se refiere a textos que abordan las distintas ER, así como su diagnóstico, tratamientos y evolución (34,5\%), distribuidas en $A B C$ (47 \%), El País (31,9 \%), La Razón (29,5 \%) y El Mundo (27,5 \%). Cabe destacar que las noticias relacionadas con esta temática, que suponen el $64,1 \%$ del total, aparecen publicadas en los meses de diciembre $(29,4 \%)$, febrero (19,4\%) y marzo (15,5\%). Algo lógico si se tiene en cuenta que en diciembre se produce el caso Nadia, en febrero se celebra el Día Mundial de las Enfermedades Raras y en marzo el caso de los 2000 tumores. En cuanto a las fuentes utilizadas en los diarios, alcanzan porcentajes elevados las que proceden de la familia (10,2 \%) (La Razón 11,1 \%, El Mundo 27,7 \%, El País 26,2 \% y ABC 43,6 \%) y las científicas con 22 piezas (La Razón 22,2 \%, El Mundo 27,7 \%, El País 26,6 \% y ABC 5,1 \%). Algunas noticias de esta temática son "Cinco nuevas enfermedades raras cada semana” ( $\mathrm{La}$ Razón 28/02/2017), "Cuando no puedes evitar autolesionarte” (El Mundo 27/02/2017), "Sanfilippo: la lucha contra un Alzheimer con cara de niño" (El País 28/02/2017) y "Las más de 6.000 enfermedades raras, ante el reto de hacerse más visibles” ( $A B C$ 25/02/2017). 


\section{Investigación}

Las piezas de esta temática (22\%) incluyen los resultados de investigaciones científicas o ensayos clínicos relacionadas con las ER que pueden suponer mejoras en la calidad de vida de los afectados por estas enfermedades. Ofrece mayor información El Mundo (33,5\%), seguido de La Razón (26,3 \%), El País $(19,3 \%)$ y $A B C(13,3 \%)$. En conjunto, predomina esta información en los meses de diciembre $(20,5 \%)$, febrero (15\%) y marzo (15\%). En cuanto a las fuentes utilizadas, son mayoritarias las procedentes de ámbitos científicos (El País 77,7 \%, La Razón 68,8 \%, ABC 45,5 \% y El Mundo 13,6\%). Le siguen a distancia hospitales $(10,4 \%)$ e institucionales (13,6\%). Algunas de las noticias sobre esta temática son "El encuentro nacional de enfermedades raras apuesta en Burgos por la Universidad” (La Razón 25/02/2017), "Tras años de investigación, hay avances en enfermedades raras” (El Mundo 19/12/2016), "El I+D+I de las enfermedades raras: investigación, diagnóstico e inclusión" (El País 28/02/2017), "Los millones de españoles con enfermedades raras demandan más investigación sobre sus patologías” ( $A B C$ 28/02/2017).

\section{Tribunales/justicia}

Este apartado se refiere a sentencias judiciales que dictaminan si hay o no delito en las denuncias relacionadas con el tema o procesos judiciales que se están tramitando. El diario que proporcionalmente más textos publica sobre este tema es El País (29,8\%), seguido de El Mundo (9\%), ABC (7,3\%) y La Razón (1,6\%). Unos contenidos que en conjunto predominan en los meses de diciembre $(48,8 \%)$ y enero $(26,8 \%)$, aunque hay discrepancias entre los periódicos. Las fuentes más empleadas resultan ser tribunales (39\%), policiales $(12,2 \%)$ y hospitalarias $(9,8 \%)$. Algunas noticias son "Detenidos los padres de Nadia ante las sospechas de estafa" (La Razón 08/12/2016), "El hombre de los dos mil tumores estafó 250.000 euros a más de 8.000 personas (El Mundo 17/03/2017), "La Audiencia de Lleida confirma la prisión provisional del padre de Nadia por riesgo de fuga" ( $E l$ País 28/03/2017), "El dinero estafado por los padres de Nadia podría ascender al millón de euros” ( $A B C$ 06/03/2017).

\section{Solidaridad}

En este apartado, se abordan los eventos y las actividades promovidos desde diversos ámbitos para dar visibilidad a las ER, y recoger fondos para 
la investigación o proyectos de interés. La información de cercanía de los problemas sociosanitarios de los ciudadanos puede materializarse en las ER en adhesiones a las reivindicaciones de los pacientes y a sus campañas de sensibilización (Bañón, 2007). Unas acciones que se vuelven relevantes para los medios, despiertan su interés y les dedican una amplia cobertura mediática (Cavaca et al., 2016). Una información que supone el 10,8\% del total y es recogida por $A B C$ (19,2 \%), La Razón (11,5 \%), El Mundo (10,5 \%) y El País (1\%). Destacan como mayoritarias las fuentes sin identificar (30,3\%), aunque se muestran discrepancias entre los diarios ( $\mathrm{La}$ Razón 44,4 \%, El Mundo 42,9\%), seguidas de las institucionales (31,2 \%). Estos contenidos tienen mayor atención mediática los meses de diciembre $(39,8 \%)$ y febrero $(24,2 \%)$ en todos los periódicos. Algunos ejemplos son "La Gala 'Inocente Inocente' recaudó 1.161 .660 euros para las enfermedades raras” (La Razón 29/12/2016), "Ponteenmodosmart: moda, gastronomía y deporte sostenible y solidario" (El Mundo 30/03/2017), "De Madrid a Dubái por un exoesqueleto para Álvaro" (El País 16/12/2017), "Gala inocente inocente": nuevas bromas a famosos bajo el lema 'Lo raro es no ayudar"” (ABC 05/01/2017).

\section{Asociaciones}

Otra temática de interés es la que procede de las asociaciones (5,9\%), que es recogida por El Mundo (10,6 \%), El País (5,3 \%), La Razón (4,9\%) y $A B C(3,6 \%)$. Su publicación se concentra en los meses de febrero (50\%) y diciembre $(22,2 \%)$. En cuanto a las fuentes utilizadas, son las propias asociaciones (66,6\%) ylas ER (33,3\%). Algunas noticias publicadas son "La Fundación Isabel Gemio presenta, en el Día Mundial de las Enfermedades Raras, un positivo balance” (La Razón 27/02/2017), "La Asociación de Fibrosis Quística recauda 5.800 en Castellón” (ElMundo 10/10/2016), “Las asociaciones de enfermedades raras reaccionan al caso Nadia: la sociedad no debe dejar de confiar" ( $A B C 17 / 12 / 2016)$.

\section{Recursos}

Incluimos los contenidos relacionados con las dotaciones disponibles en los centros de referencia que prestan apoyo (La Razón 16,4 \%, ABC 4,8 \%, El Mundo 4,5 \% y El País 4,2 \%). El mes de marzo concentra un mayor número de piezas informativas en todos los diarios con excepción de $E l$ 
País que lo hace en diciembre. Estas noticias proceden mayoritariamente de fuentes científicas $(28,6 \%)$ e institucionales (28,6\%). No obstante, se producen discrepancias: en La Razón predominan las científicas (50\%) y en El País las institucionales (75\%). Algunas noticias son "Una tarjeta sanitaria para menores con discapacidad o con enfermedades raras" ( $\mathrm{La}$ Razón 16/03/2017), "Una red de colaboración europea en enfermedades raras” (El Mundo 28/02/2017), "La última fábrica 'art déco' reconvertida en templo del arte” (El País 11/03/2017), “Regina Leal: 'Habrá Escuela de Pacientes para enfermedades raras'” (ABC 04/03/2017).

\section{Fallecimientos}

Esta temática tiene escasa presencia en El País (3,2 \%), La Razón (1,6 \%) y $A B C(2,4 \%)$. Algunos ejemplos son "La emotiva despedida de una madre tras la muerte de su bebé debido a una larga enfermedad" (La Razón 31/03/2017), "Muere Martin McGuinness, exjefe militar del IRA, a los 66 años” (El País 21/03/2017), "Muere Sergio Roque, el niño de Puertollano que padecía una enfermedad rara” ( $A B C 03 / 02 / 2017)$.

\section{Discusión y conclusiones}

Este artículo presenta como objetivo el análisis del tratamiento sobre las ER en los diarios de información general españoles. Su interés radica en la necesidad que existe en conocer la cobertura que reciben en la agenda diaria de los medios de comunicación y saber si esa información es considerada de interés y si responde a la similitud entre las agendas mediática y social que defiende McCombs (2014).

El análisis realizado demuestra que las ER son un tema emergente y de relevancia en la prensa nacional, dado que publican noticias todos los meses analizados (304 piezas), que sigue la misma tendencia de los resultados obtenidos por Arcos (2013), Bañón y Requena (2014) y SánchezHernández (2016). No obstante, se constata que aparece más información cuando coincide con eventos o acontecimientos especiales (SánchezHernández, 2016, p. 250), como ha sucedido con el Día Mundial de las Enfermedades Raras, el caso Nadia, y los 2000 tumores. También se aprecian discrepancias en cuanto al número de piezas informativas que publica cada 
periódico. Es decir, no existe unanimidad en los criterios de selección en cada diario, por lo que se puede interpretar que valoran de diferente modo a la hora de decidir la publicación de la noticia.

Con respecto a la procedencia de la información, predomina mayoritariamente el ámbito estatal frente al internacional. En el Estado español, las comunidades autónomas que más cobertura informativa reciben son Madrid (48 \%) y Cataluña (19\%), resultados que coinciden con los obtenidos por Sánchez-Castillo (2013, p. 78). Por tanto, existe un interés local o regional que destaca la proximidad como valor de la noticia (SánchezCastillo, 2012, p.19), de modo que la cercanía sin duda funciona, despierta el interés del público que la adquiere de forma inmediata (Bañón, Fornieles, Solves y Rius, 2011, p. 131). Es decir, cuanto peor es la enfermedad, más presencia tiene y se le da mayor cobertura según confirman los estudios sobre enfermedades realizado por Cavaca et al. (2016, p. 11). Estos resultados confirman que las ER forman parte de la agenda de los medios y con ello se da respuesta a la primera pregunta planteada.

El tratamiento que reciben las ER es eminentemente informativo y poco elaborado. Predominan las noticias descriptivas en detrimento de las de opiniones o de análisis, unos datos que siguen la misma tendencia de los obtenidos por Arcos (2013), Bañón (2007), López-Villafranca y Castillo-Esparcia (2018), Solves y Bañón (2014), Sánchez-Castillo (2012) y Sánchez-Castillo y Mercado (2014), resultados que confirman la segunda pregunta enunciada. Es decir, son textos genéricos con escasa profundización periodística sin apenas explicaciones ni opiniones sobre las ER, lo que denota poca preocupación por la elaboración de la información, y además lo corrobora que gran parte de las informaciones solo utilizan una fuente. Estos resultados pueden guardar relación con el elevado número de textos firmados por agencia, lo que supone similares contenidos en diferentes medios (Bañón y Requena, 2014) y el propio periódico (Solves y Bañón, 2014). Se trata, en general, de textos que se publican prácticamente sin ninguna intervención por parte de los periodistas que las escriben (Bañón y Requena, 2014; Gelado-Marcos, 2009). Los diarios analizados sitúan un mayor número de noticias sobre ER en la sección de sociedad, 
datos similares a los obtenidos por Sánchez-Hernández (2016), pero inferiores a los resultados de otros autores (Sánchez-Castillo, 2016; Solves y Bañón, 2014).

En cuanto a la temática de las piezas publicadas, predominan las relacionadas con la medicina/enfermedades (Sánchez-Castillo, 2016), de modo que se tratan las ER como un problema médico (Sánchez-Castillo y Mercado, 2014; Solves y Bañón, 2014). Le siguen la investigación, un campo que durante los últimos años ha adquirido gran auge en biomédica, clínica y epidemiológica y, con ello, se abren nuevas vías médicas (Palau, 2010). $\mathrm{Si}$ atendemos a la fecha de publicación, se observa en todos los diarios un incremento de noticias los meses de diciembre y marzo (79 textos). Si analizamos la temática, se corresponden a contenidos relacionados con estafas (caso Nadia y 2000 tumores). Unos contenidos que han sido tratados con cierto sensacionalismo creando una gran atención mediática, lo que demuestra que algunas enfermedades tienen menor repercusión en los medios, pero si ocurre algo se vuelven relevantes y suscitan el interés de los diarios (Cavaca et al., 2016). También se pueden reseñar aspectos negativos, como concluye Emerich et al. (2017) en su estudio sobre noticias de salud en los periódicos brasileños, que estos tienden a publicar informaciones que inciden en los aspectos negativos de las enfermedades. Además es de desatacar el interés humano como un valor en las piezas analizadas (Bañón et al., 2011; López-Villafranca y Castillo-Esparcia, 2018; Sánchez-Castillo y Mercado, 2014; Sánchez-Hernández, 2016). Otro mes de importancia es febrero, resultados que coinciden con los obtenidos por Sánchez-Hernández (2016), algo que resulta lógico dado que el día 28 de febrero se celebra el Día Mundial de las Enfermedades Raras y con tal motivo se organizan galas, exposiciones y eventos en los que participan personas relevantes (Cruz y Requena, 2013; Sánchez-Hernández, 2016).

En los diarios analizados, los temas relacionados con la medicina y la investigación suponen la mitad de los contenidos, resultados que siguen la misma tendencia de Palau (2010) y López-Villafranca y Castillo-Esparcia (2018). Incluyen contenidos sobre síntomas, patologías, diagnósticos $\mathrm{y}$ avances científicos tratados desde un punto de vista informativo y con 
escasos datos científicos que aporten conocimientos al lector. Resulta significativo que el $72,7 \%$ de las informaciones solo utiliza una fuente informativa y el 15,3 \% dos. Estos datos responden la tercera pregunta planteada, es decir, predominan las temáticas relacionadas con aspectos clave en las $\mathrm{ER}$, como es la medicina y la investigación, y vuelve a incidir en la escasa elaboración de los textos.

Los resultados del análisis reflejan que las ER están presentes en los diarios de información general españoles y la visión que transmiten a la sociedad se equipara al resto de enfermedades aportando un enfoque general. Sin embargo, serían necesarias otras investigaciones sobre el tema desde el ámbito de la comunicación, con el fin de estudiar otros encuadres oframing de las noticias sobre ER y profundizar de manera más exhaustiva o abordar otros aspectos como la investigación y los avances científicos, las salas virtuales o las estrategias de comunicación y la especialización de los profesionales y el uso de las fuentes expertas, para hacer más visibles las enfermedades, aunque es algo que no se plantea en los objetivos de este estudio.

\section{Referencias}

Adekunle, A. L. y Adnan, H. M. (2016). Communicating health: Media framing of Ebola outbreak in Nigerian newspapers. Jurnal Komunikasi, Malaysian Journal of Communication, 32(2), 362-380. DOI: 10.17576/JKMJC-2016-3202-19

Arcos, J. M. (2013). El Día Mundial de las enfermedades raras en las noticias de prensa: el ejemplo de Andalucía. Discurso y Sociedad, 7(1), 224-247. Recuperado de http://www.dissoc.org/ediciones/v07n01/DS7(1) Arcos.html

Aymé, S. y Schmidtke, J. (2007). Networking for rare diseases: A necessity for Europe. Bundesgesundheitsblatt-Gesundheitsforschung-Gesundheitsschutz, 50(12), 1477-1483. DOI: 10.1007/ s00103-007-0381-9 
Avellaneda, A, Izquierdo, M., Torrent-Farnell, J. y Ramón, J. R. (2007). Enfermedades raras: enfermedades crónicas que requieren un nuevo enfoque sociosanitario. Anales del Sistema Sanitario de Navarra, 30(2), 177-190. Recuperado de http://scielo.isciii.es/scielo.php?script=sci_arttext\&pid=S1 137-66272007000300002

Bañón, A. M. (2007). Las enfermedades raras y su representación discursiva: propuestas para un análisis crítico. Discurso \& Sociedad, 1(2), 188-229. Recuperado de https://www.researchgate.net/publication/28205801_Las_enfermedades_raras_y_su_representacion_discursiva_Propuestas_para_un_analisis_critico

Bañón, A. M.y Requena Romero, S. (2014). La representación de las enfermedades poco frecuentes en el discurso periodístico español: propuestas para el análisis. Pragmática Sociocultural, 8(2), 180-222. Recuperado dehttps://www.researchgate.net/publication/286911688_La_representacion_de_las_enfermedades_poco_frecuentes_en_el_discurso_periodistico_espanol_Propuestas_para_el_analisis

Bañón, A. M., Fornieles Alcaraz, J., Solves Almela, J. y Rius Sanchís, I. (Coords.) (2011). Desafíos y estrategias comunicativas de las enfermedades raras: la investigación médica como referente; estudio cuantitativo-discursivo de la prensa escrita impresa y digital española. Valencia, España: Centro de Investigación Biomédica en Red de Enfermedades Raras.

Bardin, L. (2002). Análisis de contenido. Madrid: Akal.

Blanco Castilla, E. y Paniagua Rojano, F. J. (2007). Periodismo, salud y calidad de vida. Contenidos y fuentes, FISEC-Estrategias, 3(8), 3-24. Recuperado de https://www.researchgate.net/publication/240631218_PERIODISMO_SALUD_Y_CALIDAD_DE_ VIDA_CONTENIDOS_Y_FUENTES

Berelson, B. (1952). Content analysis in communication research. Nueva York, EE. UU.: Hafner. 
Bruno, M. F. (2004). El rol del periodismo en el sistema de salud. Revista de la Asociación Médica Argentina, 117(1), 3-4.

Calvo Hernando, M. (2002). El periodismo científico, reto de las sociedades del siglo XXI. Comunicar: Revista Científica de Comunicación y Educación, 19, 15-18. Recuperado de https://www.redalyc.org/ articulo.oa? $\mathrm{id}=15801903$

Camacho Markina, I. (2009). La gripe A en la prensa española. Revista Latina de Comunicación Social, 64, 827-843. DOI: 10.4185/RLCS64-2009-865-827-843

Carrillo, M. V. (2004). Medicina preventiva en tiempos de crisis, basada en la gestión de la "marcaempresa". Zer: Revista de Estudios de Comunicación, 17, 67-83. Recuperado de http://www.ehu.eus/ojs/ index.php/Zer/article/view/5265

Cavaca, A. G., Emerich, T. B., Vasconcellos-Silva, P. R., dos Santos-Neto, E. T. y Oliveira, A. E. (2016). Diseases Neglected by the Media in Espírito Santo, Brazil in 2011-2012. PLoS neglected tropical diseases, 10(4), e0004662. DOI: 10.1371/journal.pntd.0004662

Cavaca, A. G., Vasconcellos-Silva, P. R., Ferreira, P.y Nunes, J. A. (2015). Between evidence and negligence: Coverage and invisibilityof health topics in the Portuguese printed media. Ciencia \& Saude Coletiva, 20(11), 3569-3580. DOI: 10.1590/1413-812320152011.18342014

Chaves Restrepo, A. P. (s. f.). El problema socialy de salud de las enfermedades raras: transitando de la invisibilidad a la visibilidad. Recuperado de https://s3.amazonaws.com/academia.edu.documents/32447882/ Las_Enfermedades_raras_en_Colombia-Transitando_de_la_ invisibilidad_a_la_visibilidad.pdf?AWSAccessKeyId=AKIAI WOWYYGZ2Y53UL3A\&Expires $=1541958966 \&$ Signature $=$ \%2F1rJ1W7AvKXvtfe4JpxhfZmZZvo \%3D\&response-contentdisposition=inline \%3B \%20filename \%3DLas_enfermedades raras_en_Colombia_Tran.pdf 
Comunicación de la Comisión al Parlamento Europeo, al Consejo, al Comité Económico y Social Europeo y al Comité de las Regiones. Las enfermedades raras: un reto para Europa. Recuperado de https:// ec.europa.eu/health/ph_threats/non_com/docs/rare_com_es.pdf

Costa Sánchez, C. (2008). Medicina y salud en la prensa: las noticias de salud en los principales diarios de Galicia. Revista Latina de Comunicación Social, 63, 15-21.DOI: 10.4185/RLCS-63-2008-750-015-021

Cruz Moya, O. y Requena Romero, S. (2013). El día de las enfermedades raras en las noticias televisivas: análisis crítico de su representación audiovisual. Discurso \& Sociedad, 7(1), 200-223. Recuperado de http://www.dissoc.org/ediciones/v07n01/DS7(1)Cruz\%20 \&\%20Requena.html

Dalley, S. E., Buunk, A. P. y Umit, T. (2009). Female body dissatisfaction after exposure to overweight and thin media images The role of body mass index and neuroticism. Personality and Individual Differences, 47(1), 47-51. DOI: 10.1016/j.paid.2009.01.044

Decisión no 1295/1999/CE del Parlamento Europeo y del Consejo, de 29 de abril de 1999, por la que se aprueba un programa de acción comunitaria sobre las enfermedades poco comunes en el marco de la acción en el ámbito de la salud pública (1999-2003).

Díaz Aledo, L. (2007). La representación de la discapacidad en los medios de comunicación: cómo lograr una presencia más adecuada. Comunicación e Ciudadanía, 1, 289-306.

Emerich, T. B., Cavaca, A. G., Santos-Neto, E. T., Gentilli, V. I. y Oliveira, A. E. (2017). Media valuations of health journalism and health dynamics in brazilian print media. International Journal of Social Scien e Studies, 5(1), 31-42. DOI: 10.11114/ijsss.v5i1.1997 
Fernandez, S. y Pritchard, M. (2012). Relationships between self-esteem, media influence and drive for thinness. Eating Behaviors, 13(4), 321-325. DOI: 10.1016/j.eatbeh.2012.05.004

Férriz Núñez, M (2008, abril). Desarrollo de destrezas comunicativas no verbales en clases de L1 para alumnos con síndrome de Guilles de la Tourette. Trabajo presentado en XXVI Congreso Internacional: "De la lingüística aplicada a la lingüística de la mente: Hitos, prácticas y tendencias", Universidad de Almería, Almería.

Fornieles Alcaraz, J. y Bañón Hernández, A. M. (2011). Análisis del discurso multimodal: las fotografías de la información sobre enfermedades raras. En A. M. Bañón Hernández, J. Fornieles Alcaraz, J. Solves Almela e I. Rius Sanchís (Coords.), Desafíos y estrategias comunicativas de las enfermedades raras: la investigación médica como referente; estudio cuantitativo-discursivo de la prensa escrita impresa y digital española (2009-2010) (pp. 189-200). Valencia, España: Centro de Investigación Biomédica en Red de Enfermedades Raras.

García Ribes, M., Verde González, M. P., Suerio Justel, J., Rosado Bartolomé, A. y Sorlí Guerola, J. V. (2013). Abordando las enfermedades raras desde la consulta de atención primaria: si se quiere, se puede. Revista Clínica de Medicina de Familia, 6(1), 32-36. DOI: 10.4321/ S1699-695X2013000100006

García Sánchez, J. E. y García Sánchez, E. (2005). Enfermedades raras en el cine. Revista de Medicina y Cine, 1(4), 93-94. Recuperado de http://revistas.usal.es/index.php/medicina_y_cine/article/ view/205/374

Gelado-Marcos, R. (2009). La dependencia de la prensa española hacia las agencias de noticias. Comunicación y Sociedad, 22(2), 243275.

González Borjas, A. (2004). Salud, información periodística en alza. Ámbitos: Revista internacional de comunicación, 11-12, 301-310. 
Guzmán do Nascimento, B. y Rodríguez Díaz, R. (2016). Comunicación y salud: la anorexia a través de la prensa. Estudios sobre el Mensaje Periodístico, 22(2), 747-758. DOI: 10.5209/ESMP.54233

Holsti, R. (1969). Content analysis for the social sciences and humanities. Reading, EE. UU.: Addison-Wesley.

Huerta Rodríguez, B., Fernández Sastre, B., Gallardo Hidalgo, C. y Hernández Lozano, D. (2012). El rol del trabajador social en la atención de las personas afectadas por enfermedades raras: la enfermedad de Huntington. Documentos de trabajo social: Revista de trabajo y acción social, 51, 243-267.

Huertas Roig, A. y Xifra Triadú, J. (2009). ¿Marcas o genéricos? La comunicación en línea de las marcas farmacéuticas. Zer: Revista de Estudios de Comunicación, 14(27), 251-270.

Igartua, J. J. (2006). Métodos cuantitativos de investigación en comunicación. Barcelona, España: Bosch.

Igartua, J. J. y Lopes, O. (2002). La prevención del sida mediante cortos de ficción: una investigación experimental. Zer: Revista de Estudios de Comunicación, 7(13), 115-131.

Jacobs, R., Boyd, L., Brennan, K., Sinha, C. K. y Giuliani, S. (2016). The importance of social media for patients and families affected by congenital anomalies: A Facebook cross-sectional analysis and user survey. Journal of Pediatric Surgery, 51(11), 1766-1771. DOI: 10.1016/j.jpedsurg.2016.07.008

Krippendorff, K. (1980). Content analysis: An introduction to its methodology. Beverly Hills, EE. UU.: Sage.

Landis, J. R. y Koch, G. G. (1977). The measurement of observer agreement for categorical data. Biometrics, 33, 159-174. 
López-Villafranca, P. (2016). Estudio de la presencia de los gabinetes de comunicación en las organizaciones de pacientes con enfermedades raras en España. Revista Internacional de Relaciones Públicas, 11(6), 27-46. DOI: 10.5783/RIRP-11-2016-03-27-46

López-Villafranca, P. y Castillo-Esparcia, A. (2018). El encuadre de las enfermedades raras en los medios de comunicación españoles. $O b$ servatorio $\left(\mathrm{OBS}^{*}\right), 12(2), 136-155$.

Lugones Botell, M. y Ramírez Bermúdez, M. (2012). Enfermedades raras. Revista Cubana de Medicina General Integral, 28(3). Recuperado de http://scielo.sld.cu/scielo.php?pid=S0864$21252012000300014 \&$ script=sci_arttext\&tlng=pt

Moreno Espinosa, P. (2010). Periodismo biomédico: nuevos contenidos mediáticos. Estudios sobre el Mensaje Periodístico, 16, 319-330.

McCombs, M. (2014). Setting the agenda: Mass media and public opinion. Malden, EE. UU.: Polity.

Mosquera, M. (2002). Comunicación en salud: conceptos, teorías y experiencias. Recuperado de http://www.portalcomunicacion.com/obregon/pdf/mosquera_2003.pdf

Muñoz, M., Pérez-Santos, E., Crespo, M., Guillén, A. I. y Izquierdo, S. (2011). La enfermedad mental en los medios de comunicación: un estudio empírico en prensa escrita, radio y televisión. Clínica y Salud, 22(2), 157-173. DOI: 10.5093/cl2011v22n2a5

Navarro Ruiz, C. (Dir.) (2004). La imagen de la discapacidad en la prensa de Castilla y León. Junta de Castilla y León.

Palau, F. (2010). Enfermedades raras, un paradigma emergente en la medicina del siglo XXI. Medicina Clínica, 134(4), 161-168. DOI: 10.1016/j.medcli.2009.06.038 
Peñafiel Saiz, C., Camacho Marquina, I., Aiestaran Yarza, A., Ronco López, M. M. y Echegaray-Eizaguirre, L. (2014). La divulgación de la información de salud: un reto de confianza entre los diferentes sectores implicados. Revista Latina de Comunicación Social, 69, 135-151. DOI: $10.4185 /$ RLCS-2014-1005

Pindado, J. y Aguilera Moyano, M. de (2006). Nuevos enfoques en comunicación y salud: perspectivas de investigación. Comunicar: Revista científica iberoamericana de comunicación y educación, 26, 13-20.

Piñuel Raigada, J. L. y Gaitán Moya, J. A. (1995). Metodología general: conocimiento científico e investigación en la comunicación social. Madrid, España: Síntesis.

Ramírez Queralt, A. y Rius Sanchís, I. (2010). Valores diferenciales de las enfermedades raras: denuncia, reivindicación y drama. En A. M. Bañón Hernández, J. Fornieles Alcaraz, J. Solves Almela e I. Rius Sanchís (Coords.), Desafíos y estrategias comunicativas de las enfermedades raras: la investigación médica como referente; estudio cuantitativo-discursivo de la prensa escrita impresa y digital española (2009-2010) (pp. 127-150). Valencia, España: Centro de Investigación Biomédica en Red de Enfermedades Raras.

Regulation (EC) No 141/2000 of the European Parliament and of the Council of 16 December 1999 on orphan medicinal products.

Revuelta, G. (2006). Salud y medios de comunicación en España. Gaceta Sanitaria, 20, 203-208.

Revuelta, G., De Semir, V. y Minelli de Oliveira, J. (1997). Medicina y salud en la prensa diaria: informe Quiral 1997-2006. Observatorio de la Comunicación Científica/Universitat Pompeu Fabra.

Rodrigo, S. R. (2011). Ideología en prensa española: tratamiento discursivo de la enfermedad rara ataxia. Estudios sobre el Mensaje Pe- 
riodístico, 17(2), 581-594.DOI: 10.5209/rev_ESMP.2011.v17. $\mathrm{n} 2.38132$

Rius Sanchís, I. y Solves Almela, J. (2009). Discapacidad y medios de comunicación: la noticia como relato épico. En J.M. Noguera (Coord.), El drama del periodismo, narración e información en la cultura del espectáculo: actas de las conferencias y comunicaciones del XI Congreso de la Sociedad Española de Periodística (pp. 411-424). Murcia, España: Sociedad Española de Periodística.

Roberts, A. y Good, E. (2010). Media images and female body dissatisfaction: The moderating effects of the Five-Factor traits. Eating Behaviors, 11(4), 211-216. DOI: 10.1016/j.eatbeh.2010.04.002

Rodríguez Díaz, S. y Ferreira, M. (2010). Desde la dis-capacidad hacia la diversidad funcional: un ejercicio de dis-normalización. Revista Internacional de Sociología, 68(2), 289-309.

Rodríguez Muñoz, F.J. (2007). Comunicación, autismo y síndrome de Asperger: hacia un estado de la cuestión desde el punto de vista bibliográfico. Tonos Digital, 13, 1-37.

Sánchez Castillo, S. (2012). Representación social de las enfermedades raras en la prensa española. Aposta: Revista de Ciencias Sociales, 54, 1-31.

Sánchez Castillo, S. (2013). Las enfermedades raras en la prensa española: una aproximación empírica desde la teoría del framing. Ámbitos: Revista Internacional de Comunicación, 22, 71-80.

Sánchez-Castillo, S. y Mercado Sáez, M. T. (2014). El encuadre de las enfermedades raras en la prensa española. Anuario Electrónico de Estudiosen Comunicación Social, 7(1), 104-128. Recuperado de https:// dialnet.unirioja.es/servlet/articulo? codigo $=4800928$

Sánchez-Hernández, F. (2016). Análisis sobre los contenidos de enfermedades raras en la prensa escrita española. Revista Españo- 
la de Comunicación en Salud, 7(2), 242-260. DOI: 10.20318/ recs.2016.3450

Santos Diez, M. T. (2008). El auge de la prensa gratuita en España. Bilbao, España: Universidad del País Vasco.

Santos Diez, M. T. (2009). La salud en la prensa gratuita especializada: un fenómeno emergente. Estudios sobre el Mensaje Periodístico, 15, 477-490.

Santos Diez, M. T. y Pérez Dasilva, J. (2017). Las federaciones de asociaciones de enfermedades raras en Facebook y Twitter. En A. Larrondo Ureta, K. Meso Ayerdi y S. Peña Fernández (Coords.), IX Congreso Internacional de Ciberperiodismo: Innovación y emprendimiento al servicio de las audiencias (pp. 498-511). Bilbao, España: Universidad del País Vasco.

Schumacher, K. R., Stringer, K. A., Donohue, J. E., Yu, S., Shaver, A., Caruthers, R. L., ... Russell, M. W. (2014). Social media methods for studying rare diseases. Pediatrics, 133(5), 1345-1353. DOI: 10.1542/peds.2013-2966

Seco Sauces, M. O. y Ruiz-Callado, R. (2016). Las enfermedades raras en España: un enfoque social. Prisma, 17, 373-397.

Schieppati, A., Henter, J. I., Daina, E. y Aperia, A. (2008). Why rare diseases are an important medical and social issue. The Lancet, 371(9629), 2039-2041.

Solves Almela, J. y Bañón Hernández, A. M. (Coords.) (2014). Las enfermedades raras en los medios. Observatorio sobre Enfermedades Raras.

Sontag, S. (1978). Illness as metaphor. Nueva York, EE. UU.: Straus Giroux. 
Terrón Blanco, J. L. (2012). El tratamiento del VIH/sida en los periódicos españoles: una investigación colaborativa. Revista de Comunicación y Salud, 1(1), 4-17.

Torrent-Farnell, J. y Morros, R. (2001). The EU challenges on the designation of orphan medicinal products. Pharmaceuticals Policy and Law, 3, 19-30.

Westall Pixton, D. L. (2011). La obesidad infantil en la prensa española. Estudios sobre el Mensaje Periodístico, 17(1), 225-239.

Wimmer, R. y Dominick, J. (1996). La investigación científica de los medios de comunicación: una introducción a sus métodos. Barcelona, España: Bosch.

Yardley, L. (Ed.) (1997). Material discourses of health and illness. Londres, RU: Routledge.

Yoo, J. H. y Kim, J. (2012). Obesity in the new media: A content analysis of obesity videos on YouTube. Health Communication, 27(1), 86-97. 